\title{
Global and blow-up solutions for a nonlinear reaction diffusion equation with Robin boundary conditions
}

\author{
Huimin Tian ${ }^{1}$ and Lingling Zhang ${ }^{1,2^{*}}$
}

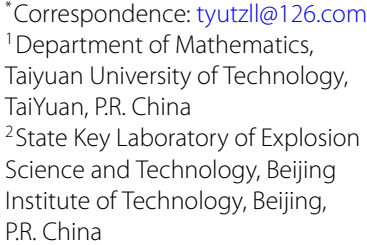

"Correspondence: tyutzll@126.com 1 Department of Mathematics, Taiyuan University of Technology, TaiYuan, P.R. China

${ }^{2}$ State Key Laboratory of Explosion Science and Technology, Beijing Institute of Technology, Beijing, P.R. China

\begin{abstract}
In the paper, we investigate global and blow-up solutions for a class of nonlinear reaction diffusion equations with Robin boundary conditions. By using auxiliary functions and a first-order differential inequality technique, we establish conditions on the data to prove the existence of global solution. Moreover, based on maximum principles, we obtain a criterion that guarantees the occurrence of the blow-up. When blow-up occurs, we discuss an upper bound and a lower bound on blow-up time. At last, we apply two examples to illustrate our main results.
\end{abstract}

Keywords: Reaction diffusion equations; Blow-up; Global; Lower and upper bounds

\section{Introduction}

In recent years, there has been a great deal of literature on the global and blow-up solutions for nonlinear partial differential equations, for instance, in [1-8]. These works have contained a lot of interesting results about the global solutions, blow-up of solutions, bounds for the blow-up time, blow-up rates, and so on. A variety of physical, chemical, and biological applications are discussed in $[9,10]$.

As far as we know, reaction-diffusion equation is an important part of partial differential equation. There have been many research papers devoted to the nonlinear reaction diffusion equations under various boundary conditions, such as Dirichlet boundary conditions $[11,12]$, Neumann boundary conditions [13-16], nonlocal boundary conditions [17, 18], and nonlinear boundary conditions $[19,20]$. At the same time, the blow-up problems for reaction diffusion equations under Robin boundary conditions have been also studied (see [21-24]). The authors in [22] considered the following equation:

$$
u_{t}=\nabla \cdot\left(\rho\left(|\nabla u|^{2}\right) \nabla u\right)+f(u), \quad(x, t) \in \Omega \times(0, T)
$$

where $\Omega \subset R^{n}(n \geq 2)$ is a smooth bounded domain. By making appropriate restrictions and using a differential inequality technique, a lower bound for the blow-up time was investigated in a three-dimensional space if blow-up occurs. When $\Omega \subset R^{n}(n \geq 2)$, the authors demonstrated a criterion which guarantees the solution to remain global. [23]

(c) The Author(s) 2020. This article is licensed under a Creative Commons Attribution 4.0 International License, which permits use sharing, adaptation, distribution and reproduction in any medium or format, as long as you give appropriate credit to the original author(s) and the source, provide a link to the Creative Commons licence, and indicate if changes were made. The images or other third party material in this article are included in the article's Creative Commons licence, unless indicated otherwise in a credit line to the material. If material is not included in the article's Creative Commons licence and your intended use is not permitted by statutory regulation or exceeds the permitted use, you will need to obtain permission directly from the copyright holder. To view a copy of this licence, visit http://creativecommons.org/licenses/by/4.0/. 
was concerned with a more complicated case:

$$
(g(u))_{t}=\nabla \cdot\left(\rho\left(|\nabla u|^{2}\right) \nabla u\right)+k(t) f(u), \quad(x, t) \in \Omega \times(0, T) .
$$

According to a differential inequality technique and maximum principles, the authors showed a blow-up or non-blow-up criterion under some appropriate assumptions. Moreover, they were dedicated to the upper and lower bounds for the blow-up time when blowup occurs. In [24], the authors dealt with the blow-up phenomena of the following quasilinear reaction diffusion equations with weighted nonlocal source:

$$
(g(u))_{t}=\nabla \cdot\left(\rho\left(|\nabla u|^{2}\right) \nabla u\right)+a(x) f(u), \quad(x, t) \in \Omega \times(0, T),
$$

where $\Omega \subset R^{n}(n \geq 2)$ is a bounded convex domain. They established conditions to guarantee that the solution remains global or blows up in a finite time. Moreover, an upper and lower bounds for blow-up time were derived. These results were obtained by utilizing a differential inequality technique on suitably defined auxiliary functions.

Inspired by the above papers, we are concerned with the following reaction diffusion equations under Robin boundary conditions:

$$
\begin{cases}(g(u))_{t}=\nabla \cdot\left(\rho\left(|\nabla u|^{p}\right) \nabla u\right)+k(t) a(x) f(u), & (x, t) \in \Omega \times(0, T), \\ \frac{\partial u}{\partial \nu}+\gamma u=0, & (x, t) \in \partial \Omega \times(0, T), \\ u(x, 0)=u_{0}(x), & x \in \bar{\Omega} .\end{cases}
$$

Here, $p>0$ and $\Omega \subset \mathbb{R}^{n}(n>2)$ is a bounded convex domain and the boundary $\partial \Omega$ is smooth. $\bar{\Omega}$ is the closure of $\Omega \cdot \frac{\partial u}{\partial v}$ stands for the outward normal derivative on $\partial \Omega, \gamma$ is a positive constant, $u_{0}$ denotes the initial value and is a positive $C^{2}(\bar{\Omega})$ function, $T$ is the blow-up time if the blow-up happens, otherwise $T=+\infty$. Set $R^{+}=(0,+\infty), g$ is a $C^{2}\left(R^{+}\right)$ function with $g^{\prime}(s)>0$ for all $s>0, \rho$ is a positive $C^{2}\left(\overline{R^{+}}\right)$function with $\rho(s)+p s \rho^{\prime}(s)>0$ for $s>0, k$ is a positive $C^{1}\left(R^{+}\right)$function, $a$ is a positive $C^{1}(\bar{\Omega})$ function, $f$ is a nonnegative $C^{1}\left(R^{+}\right)$function. The maximum principles imply that the classical solution $u$ of (1.1) is a positive solution in $\bar{\Omega} \times[0, T)$.

It is obvious that problem (1.1) is more general than the ones in [22-24]. The purpose of the paper is to get a non-blow-up or blow-up criterion for system (1.1). We need to define appropriate auxiliary functions that are different from the ones in [22-24]. By using first-order differential inequalities, we show that the solution of (1.1) exists globally under some conditions. Furthermore, combining the first-order differential inequalities with maximum principles, we demonstrate that the solution $u(x, t)$ blows up at some finite time. When blow-up does occur, the upper and lower bounds for the blow-up time are presented.

The rest of the paper is organized as follows. Section 2 shows that solution $u(x, t)$ of $(1.1)$ exists globally in the suitable measure. In Sect. 3, we prove that solution $u(x, t)$ blows up at some finite time and obtain an upper bound for the blow-up time. Section 4 presents a lower bound for the blow-up time when blow-up occurs. The last section gives two examples to illustrate our main results. 


\section{Global existence}

In the section, under some suitable conditions, we prove the existence of global solution $u$ of problem (1.1). We suppose that $u$ is a classical solution of problem (1.1). In order to obtain our main result, it is required to construct the following notations and functions:

$$
\begin{aligned}
& F(s):=\int_{0}^{s} f(y) \mathrm{d} y, \quad G(s):=2 \int_{0}^{s} y g^{\prime}(y) \mathrm{d} y, \quad \Phi(t):=\int_{\Omega} G(u(x, t)) \mathrm{d} x, \\
& \Psi(t):=-\gamma \int_{\partial \Omega} u^{2} \mathrm{~d} S-\int_{\Omega}|\nabla u|^{2} \mathrm{~d} x+2 k(t) \int_{\Omega} a(x) F(u) \mathrm{d} x .
\end{aligned}
$$

Throughout the paper, we need to assume that $\lambda$ is the first positive eigenvalue of the following problem:

$$
\begin{cases}\Delta \omega+\lambda \omega=0, & x \in \Omega, \\ \frac{\partial \omega}{\partial \nu}+\gamma \omega=0, & x \in \partial \Omega,\end{cases}
$$

where

$$
0<\gamma<\frac{\lambda L_{0}}{\mu n(p q+1)}, \quad L_{0}=\min _{\partial \Omega}(x \cdot v), \mu \geq 1 .
$$

In what follows, the main result will be stated.

Theorem 2.1 Let u be a classical solution of problem (1.1). And the following assumptions on the functions $\rho, g, a, f$ hold:

$$
\begin{aligned}
& a(x) f(s(x, t)) \leq a_{1}(s(x, t))^{\kappa}\left(\int_{\Omega}(s(x, t))^{l} \mathrm{~d} x\right)^{m}, \\
& \rho(s) \geq b_{1}+b_{2} s^{2 q}, \quad g^{\prime}(s) \leq c, \quad s>0,
\end{aligned}
$$

where $l, m, \kappa, a_{1}, q, c, b_{1}, b_{2}$ are positive constants satisfying

$$
\kappa>1, \quad 0<l<\kappa+1<2 p q+2, \quad \kappa+\operatorname{lm}<2 p q+1 .
$$

Then $u$ cannot blow up in the measure $\Phi(t)$ for all time $t>0$, that is, there exists a global solution for problem (1.1) in the measure $\Phi(t)$.

Proof Taking the derivation the function $\Phi(t)$ in (2.1), we get

$$
\begin{aligned}
\Phi^{\prime}(t) & =\int_{\Omega} G^{\prime}(u(x, t)) u_{t} \mathrm{~d} x \\
& =2 \int_{\Omega} u\left(\nabla \cdot\left(\rho\left(|\nabla u|^{p}\right) \nabla u\right)+k(t) a(x) f(u)\right) \mathrm{d} x \\
& =2 \int_{\Omega} \nabla \cdot\left(u \rho\left(|\nabla u|^{p}\right) \nabla u\right)-\rho\left(|\nabla u|^{p}\right)|\nabla u|^{2}+k(t) a(x) f(u) u \mathrm{~d} x . \\
& =2 \int_{\partial \Omega} u \rho\left(|\nabla u|^{p}\right) \frac{\partial u}{\partial \nu} \mathrm{d} S-2 \int_{\Omega} \rho\left(|\nabla u|^{p}\right)|\nabla u|^{2} \mathrm{~d} x+2 \int_{\Omega} k(t) a(x) f(u) u \mathrm{~d} x
\end{aligned}
$$




$$
\begin{aligned}
= & -2 \gamma \int_{\partial \Omega} u^{2} \rho\left(|\nabla u|^{p}\right) \mathrm{d} S-2 \int_{\Omega} \rho\left(|\nabla u|^{p}\right)|\nabla u|^{2} \mathrm{~d} x \\
& +2 \int_{\Omega} k(t) a(x) f(u) u \mathrm{~d} x,
\end{aligned}
$$

where the divergence theorem and Robin boundary condition in (1.1) are used. Furthermore, conditions (2.3) and (2.4) imply that

$$
\begin{aligned}
\Phi^{\prime}(t) \leq & -2 \gamma \int_{\partial \Omega} u^{2} \rho\left(|\nabla u|^{p}\right) \mathrm{d} S-2 \int_{\Omega}\left(b_{1}+b_{2}|\nabla u|^{2 p q}\right)|\nabla u|^{2} \mathrm{~d} x \\
& +2 k(t) \int_{\Omega} a_{1} u^{\kappa+1} \mathrm{~d} x\left(\int_{\Omega} u^{l} \mathrm{~d} x\right)^{m} \\
\leq & -2 b_{2} \int_{\Omega}|\nabla u|^{2 p q+2} \mathrm{~d} x+2 k(t) \int_{\Omega} a_{1} u^{\kappa+1} \mathrm{~d} x\left(\int_{\Omega} u^{l} \mathrm{~d} x\right)^{m} .
\end{aligned}
$$

Next, we deal with the first term on the right-hand side of (2.7). By virtue of Hölder's inequality, we have the following inequality:

$$
\begin{aligned}
\int_{\Omega}\left|\nabla\left(u^{p q+1}\right)\right|^{2} \mathrm{~d} x & =(p q+1)^{2} \int_{\Omega} u^{2 p q}|\nabla u|^{2} \mathrm{~d} x \\
& \leq(p q+1)^{2}\left(\int_{\Omega} u^{2 p q+2} \mathrm{~d} x\right)^{\frac{p q}{p q+1}}\left(\int_{\Omega}|\nabla u|^{2 p q+2} \mathrm{~d} x\right)^{\frac{1}{p q+1}} .
\end{aligned}
$$

The general Poincaré inequality yields

$$
\begin{aligned}
\lambda \int_{\Omega} u^{2 p q+2} \mathrm{~d} x & \leq \int_{\Omega}\left|\nabla\left(u^{p q+1}\right)\right|^{2} \mathrm{~d} x-\int_{\partial \Omega} u^{p q+1} \frac{\partial u^{p q+1}}{\partial v} \mathrm{~d} S \\
& =\int_{\Omega}\left|\nabla\left(u^{p q+1}\right)\right|^{2} \mathrm{~d} x+\gamma(p q+1) \int_{\partial \Omega} u^{2 p q+2} \mathrm{~d} S
\end{aligned}
$$

where the Robin boundary conditions in (1.1) are used. We apply the divergence theorem to derive

$$
\begin{aligned}
\int_{\partial \Omega} u^{2 p q+2}(x \cdot v) \mathrm{d} S & =\int_{\Omega} \nabla \cdot\left(u^{2 p q+2} x\right) \mathrm{d} x \\
& =n \int_{\Omega} u^{2 p q+2} \mathrm{~d} x+2(p q+1) \int_{\Omega} u^{2 p q+1}(\nabla u \cdot x) \mathrm{d} x \\
& \leq n \int_{\Omega} u^{2 p q+2} \mathrm{~d} x+2 d(p q+1) \int_{\Omega} u^{2 p q+1}|\nabla u| \mathrm{d} x .
\end{aligned}
$$

Then it follows from Hölder's inequality and Young's inequality that

$$
\begin{aligned}
\int_{\partial \Omega} u^{2 p q+2} \mathrm{~d} S & \leq \frac{n}{L_{0}} \int_{\Omega} u^{2 p q+2} \mathrm{~d} x+\frac{2 d(p q+1)}{L_{0}} \int_{\Omega} u^{2 p q+1}|\nabla u| \mathrm{d} x \\
& =\frac{n}{L_{0}} \int_{\Omega} u^{2 p q+2} \mathrm{~d} x+\frac{2 d}{L_{0}} \int_{\Omega} u^{p q+1}\left|\nabla\left(u^{p q+1}\right)\right| \mathrm{d} x \\
& \leq \frac{n}{L_{0}} \int_{\Omega} u^{2 p q+2} \mathrm{~d} x+2\left(\left(\frac{d}{L_{0}}\right)^{2} \theta \int_{\Omega} u^{2 p q+2} \mathrm{~d} x\right)^{\frac{1}{2}}
\end{aligned}
$$


Than and Chang Boundary Value Problems

(2020) $2020: 68$

Page 5 of 19

$$
\begin{aligned}
& \times\left(\int_{\Omega} \theta^{-1}\left|\nabla\left(u^{p q+1}\right)\right|^{2} \mathrm{~d} x\right)^{\frac{1}{2}} \\
\leq & \left(\frac{n}{L_{0}}+\left(\frac{d}{L_{0}}\right)^{2} \theta\right) \int_{\Omega} u^{2 p q+2} \mathrm{~d} x+\theta^{-1} \int_{\Omega}\left|\nabla\left(u^{p q+1}\right)\right|^{2} \mathrm{~d} x,
\end{aligned}
$$

where

$$
\theta=\frac{\lambda L_{0}^{2}-\mu \gamma(p q+1) n L_{0}}{\mu \gamma(p q+1) d^{2}}>0, \quad d=\max _{\bar{\Omega}}|x| .
$$

After inserting (2.10) into (2.9), we obtain

$$
\int_{\Omega}\left|\nabla\left(u^{p q+1}\right)\right|^{2} \mathrm{~d} x \geq M_{0} \int_{\Omega} u^{2 p q+2} \mathrm{~d} x
$$

Here

$$
M_{0}=\frac{\lambda-\gamma(p q+1)\left[\frac{n}{L_{0}}+\left(\frac{d}{L_{0}}\right)^{2} \theta\right]}{1+\gamma(p q+1) \theta^{-1}}>0 .
$$

We substitute (2.12) into (2.8) to get

$$
\int_{\Omega}|\nabla u|^{2 p q+2} \mathrm{~d} x \geq \frac{M_{0}^{p q+1}}{(p q+1)^{2 p q+2}} \int_{\Omega} u^{2 p q+2} \mathrm{~d} x
$$

Therefore, (2.7) can be rewritten as follows:

$$
\Phi^{\prime}(t) \leq-\frac{2 b_{2} M_{0}^{p q+1}}{(p q+1)^{2(p q+1)}} \int_{\Omega} u^{2 p q+2} \mathrm{~d} x+2 k(t) \int_{\Omega} a_{1} u^{\kappa+1} \mathrm{~d} x\left(\int_{\Omega} u^{l} \mathrm{~d} x\right)^{m}
$$

From Hölder's inequality, we deduce

$$
\begin{aligned}
& \int_{\Omega} u^{l} \mathrm{~d} x \leq\left(\int_{\Omega} u^{\kappa+1} \mathrm{~d} x\right)^{\frac{l}{\kappa+1}}|\Omega|^{\frac{\kappa+1-l}{\kappa+1}}, \\
& \int_{\Omega} u^{\kappa+1} \mathrm{~d} x \leq\left(\int_{\Omega} u^{2 p q+2} \mathrm{~d} x\right)^{\frac{\kappa+1}{2 p q+2}}|\Omega|^{\frac{2 p q-\kappa+1}{2 p q+2}} .
\end{aligned}
$$

Obviously, the following inequality holds:

$$
\int_{\Omega} u^{2 p q+2} \mathrm{~d} x \geq\left(\int_{\Omega} u^{\kappa+1} \mathrm{~d} x\right)^{\frac{2 p q+2}{\kappa+1}}|\Omega|^{-\frac{2 p q-\kappa+1}{\kappa+1}} .
$$

Combining (2.13) and (2.14)-(2.16), we derive

$$
\begin{aligned}
\Phi^{\prime}(t) \leq & 2 k(t)\left(\int_{\Omega} u^{\kappa+1} \mathrm{~d} x\right)^{1+\frac{l m}{\kappa+1}}|\Omega|^{\frac{(\kappa+1-l) m}{\kappa+1}}\left\{a_{1}-\frac{b_{2} M_{0}^{p q+1}}{k(t)(p q+1)^{2 p q+2}}\right. \\
& \left.\times|\Omega|^{-\frac{2 p q-\kappa-l m+1}{\kappa+1}-m}\left(\int_{\Omega} u^{\kappa+1} \mathrm{~d} x\right)^{\frac{2 p q+2-l m}{\kappa+1}-1}\right\} .
\end{aligned}
$$


An application of Hölder's inequality implies

$$
\int_{\Omega} u^{2} \mathrm{~d} x \leq\left(\int_{\Omega} u^{\kappa+1} \mathrm{~d} x\right)^{\frac{2}{\kappa+1}}|\Omega|^{\frac{\kappa-1}{\kappa+1}}
$$

that is,

$$
\int_{\Omega} u^{\kappa+1} \mathrm{~d} x \geq\left(\int_{\Omega} u^{2} \mathrm{~d} x\right)^{\frac{\kappa+1}{2}}|\Omega|^{\frac{1-\kappa}{2}}
$$

Consequently, (2.17) can be replaced by

$$
\begin{aligned}
\Phi^{\prime}(t) \leq & 2 k(t)\left(\int_{\Omega} u^{\kappa+1} \mathrm{~d} x\right)^{1+\frac{l m}{\kappa+1}}|\Omega|^{\frac{(\kappa+1-l) m}{\kappa+1}}\left\{a_{1}-\frac{b_{2} M_{0}^{p q+1}}{k(t)(p q+1)^{2 p q+2}}\right. \\
& \left.\times|\Omega|^{-\frac{2 p q+1-\kappa-l m}{2}-m}\left(\int_{\Omega} u^{2} \mathrm{~d} x\right)^{\frac{2 p q+1-\kappa-l m}{2}}\right\} .
\end{aligned}
$$

Assumptions (2.1) and (2.4) imply

$$
G(u)=2 \int_{0}^{u} y g^{\prime}(y) \mathrm{d} y \leq 2 c \int_{0}^{u} y \mathrm{~d} y=c u^{2} .
$$

Hence,

$$
u^{2} \geq \frac{1}{c} G(u)
$$

It follows from (2.19) and (2.20) that

$$
\begin{aligned}
\Phi^{\prime}(t) \leq & 2 k(t)\left(\int_{\Omega} u^{\kappa+1} \mathrm{~d} x\right)^{1+\frac{l m}{\kappa+1}}|\Omega|^{\frac{(\kappa+1-l) m}{\kappa+1}}\left\{a_{1}-\frac{b_{2} M_{0}^{p q+1}}{k(t)(p q+1)^{2 p q+2}}\right. \\
& \left.\times|\Omega|^{-\frac{2 p q+1-\kappa-l m}{2}-m}\left(\int_{\Omega} \frac{1}{c} G(u) \mathrm{d} x\right)^{\frac{2 p q+1-\kappa-l m}{2}}\right\} \\
= & 2 k(t)\left(\int_{\Omega} u^{\kappa+1} \mathrm{~d} x\right)^{1+\frac{l m}{\kappa+1}}|\Omega|^{\frac{(\kappa+1-l) m}{\kappa+1}}\left\{a_{1}-\frac{b_{2} M_{0}^{p q+1}}{(p q+1)^{2 p q+2} k(t) c^{\frac{2 p q+1-\kappa-l m}{2}}}\right. \\
& \left.\times|\Omega|^{-\frac{2 p q+1-\kappa-l m}{2}-m} \Phi(t)^{\frac{2 p q+1-\kappa-l m}{2}}\right\} .
\end{aligned}
$$

From (2.21), we are devoted to proving that $u$ cannot blow up in the measure $\Phi(t)$. Now, suppose that $u$ blows up at some finite $t^{*}$ in the measure $\Phi(t)$. Then we could find

$$
\lim _{t \rightarrow t^{*-}} k(t)=k\left(t^{*}\right), \quad \lim _{t \rightarrow t^{*-}} \Phi(t)=+\infty .
$$

Moreover,

$$
\lim _{t \rightarrow t^{*-}} a_{1}-\frac{b_{2} M_{0}^{p q+1}}{(p q+1)^{2 p q+2} k(t) c^{\frac{2 p q+1-\kappa-l m}{2}}}|\Omega|^{-\frac{2 p q+1-\kappa-l m}{2}-m} \Phi(t)^{\frac{2 p q+1-\kappa-l m}{2}}=-\infty .
$$


Therefore, (2.21) yields that there exists $t_{1}<t^{*}$ such that $\Phi^{\prime}(t)<0, t \in\left(t_{1}, t^{*}\right)$. Namely,

$$
\Phi\left(t_{1}\right)>\Phi(t), \quad t \in\left(t_{1}, t^{*}\right)
$$

We take the limit as $t \rightarrow t^{*-}$ to get

$$
\Phi\left(t_{1}\right) \geq \lim _{t \rightarrow t^{*-}} \Phi(t)=+\infty
$$

This is a contradiction. Then the hypothesis that $u$ blows up at some finite time is false.

\section{Blow-up in finite time and an upper bound for blow-up time}

This subsection presents that $u$ blows up in finite time and derives an upper bound for blow-up time under some suitable assumptions of the functions $\rho, b, k, f, g$. Firstly, we state the following Lemma 3.1 which is used for the proof of the main result.

Lemma 3.1 Suppose that $u$ is a classical solution of problem (1.1). And the following assumptions are satisfied:

$$
\begin{aligned}
& k^{\prime}(t) \geq 0, \quad t>0 . \\
& \nabla \cdot\left(\rho\left(\left|\nabla u_{0}\right|^{p}\right) \nabla u_{0}\right)+k(0) a(x) f\left(u_{0}\right) \geq 0, \quad x \in \bar{\Omega} .
\end{aligned}
$$

Then

$$
u_{t}(x, t) \geq 0, \quad(t, x) \in\left[0, t^{*}\right) \times \bar{\Omega} .
$$

Proof Taking a derivative of $u_{t}$ with respect to $t$, we have

$$
\begin{aligned}
u_{t t}= & \left(\frac{1}{g^{\prime}(u)}\left(\nabla \cdot\left(\rho\left(|\nabla u|^{p}\right) \nabla u\right)+k(t) a(x) f(u)\right)\right)_{t} \\
= & \left(\frac{1}{g^{\prime}}\left(\rho \Delta u+p \rho^{\prime}|\nabla u|^{p-2} \sum_{i, j=1}^{n} u_{x_{i}} u_{x_{j}} u_{x_{i} x_{j}}+k(t) a(x) f(u)\right)\right)_{t} \\
= & \frac{\rho}{g^{\prime}} \Delta u_{t}+\frac{p \rho^{\prime} g^{\prime}|\nabla u|^{p-2} \nabla u \cdot \nabla u_{t}-\rho g^{\prime \prime} u_{t}}{g^{2}} \Delta u \\
& +\frac{g^{\prime} p^{2} \rho^{\prime \prime}|\nabla u|^{p-2} \nabla u \cdot \nabla u_{t}-p \rho^{\prime} g^{\prime \prime} u_{t}}{g^{\prime 2}}|\nabla u|^{p-2} \sum_{i, j=1}^{n} u_{x_{i}} u_{x_{j}} u_{x_{i} x_{j}} \\
& +\frac{p \rho^{\prime}(p-2)|\nabla u|^{p-4} \nabla u \cdot \nabla u_{t}}{g^{\prime}} \sum_{i, j=1}^{n} u_{x_{i}} u_{x_{j}} u_{x_{i} x_{j}} \\
& +\frac{2 p \rho^{\prime}|\nabla u|^{p-2}}{g^{\prime}} \sum_{i, j=1}^{n}\left(u_{t}\right)_{x_{i}} u_{x_{j}} u_{x_{i} x_{j}} \\
& +\frac{\left(a k^{\prime} f+a k f^{\prime} u_{t}\right) g^{\prime}-g^{\prime \prime} u_{t} a k f}{g^{\prime 2}}+\frac{p \rho^{\prime}|\nabla u|^{p-2}}{g^{\prime}} \sum_{i, j=1}^{n} u_{x_{i}} u_{x_{j}}\left(u_{t}\right)_{x_{i} x_{j}} .
\end{aligned}
$$


For convenience, let $\xi=u_{t}$, then we get

$$
\begin{aligned}
\xi_{t}= & \frac{\rho}{g^{\prime}} \Delta \xi+\frac{p \rho^{\prime}|\nabla u|^{p-2}}{g^{\prime}} \sum_{i, j=1}^{n} u_{x_{i}} u_{x_{j}} \xi_{x_{i} x_{j}}+\left(\frac{p \rho^{\prime}|\nabla u|^{p-2} \Delta u}{g^{\prime}}+\frac{\rho^{\prime \prime} p^{2}|\nabla u|^{2 p-4}}{g^{\prime}}\right. \\
& \left.\times \sum_{i, j=1}^{n} u_{x_{i}} u_{x_{j}} u_{x_{i} x_{j}}+\frac{p(p-2) \rho^{\prime}|\nabla u|^{p-4}}{g^{\prime}} \sum_{i, j=1}^{n} u_{x_{i}} u_{x_{j}} u_{x_{i} x_{j}}\right) \nabla u \cdot \nabla \xi \\
& +\frac{2 p \rho^{\prime}|\nabla u|^{p-2}}{g^{\prime}} \sum_{i, j=1}^{n} u_{x_{j}} u_{x_{i} x_{j}} \xi_{x_{i}}+\left(\frac{a k f^{\prime}}{g^{\prime}}-\frac{g^{\prime \prime} a k f}{g^{\prime 2}}\right. \\
& \left.-\frac{\rho g^{\prime \prime}}{g^{\prime 2}} \Delta u-\frac{p \rho^{\prime} g^{\prime \prime}|\nabla u|^{p-2}}{g^{\prime 2}} \sum_{i, j=1}^{n} u_{x_{i}} u_{x_{j}} u_{x_{i} x_{j}}\right) \xi+\frac{a k^{\prime} f}{g^{\prime}} .
\end{aligned}
$$

By (3.1) and the properties of the functions $a, f, g$ for any $(t, x) \in\left[0, t^{*}\right) \times \bar{\Omega}$, we derive

$$
\begin{aligned}
\frac{\rho}{g^{\prime}} \Delta \xi & +\frac{p \rho^{\prime}|\nabla u|^{p-2}}{g^{\prime}} \sum_{i, j=1}^{n} u_{x_{i}} u_{x_{j}} \xi_{x_{i} x_{j}}+\left(\frac{p \rho^{\prime}|\nabla u|^{p-2} \Delta u}{g^{\prime}}+\frac{\rho^{\prime \prime} p^{2}|\nabla u|^{2 p-4}}{g^{\prime}}\right. \\
& \left.\times \sum_{i, j=1}^{n} u_{x_{i}} u_{x_{j}} u_{x_{i} x_{j}}+\frac{p(p-2) \rho^{\prime}|\nabla u|^{p-4}}{g^{\prime}} \sum_{i, j=1}^{n} u_{x_{i}} u_{x_{j}} u_{x_{i} x_{j}}\right) \nabla u \cdot \nabla \xi \\
& +\frac{2 p \rho^{\prime}|\nabla u|^{p-2}}{g^{\prime}} \sum_{i, j=1}^{n} u_{x_{j}} u_{x_{i} x_{j}} \xi_{x_{i}}+\left(\frac{a k f^{\prime}}{g^{\prime}}-\frac{g^{\prime \prime} a k f}{g^{\prime 2}}\right. \\
& \left.-\frac{\rho g^{\prime \prime}}{g^{\prime 2}} \Delta u-\frac{p \rho^{\prime} g^{\prime \prime}|\nabla u|^{p-2}}{g^{\prime 2}} \sum_{i, j=1}^{n} u_{x_{i}} u_{x_{j}} u_{x_{i} x_{j}}\right) \xi-\xi_{t} \\
= & -\frac{a k^{\prime} f}{g^{\prime}} \leq 0 .
\end{aligned}
$$

The Robin boundary condition of (1.1) implies

$$
\frac{\partial \xi}{\partial v}=\frac{\partial u_{t}}{\partial v}=\left(\frac{\partial u}{\partial v}\right)_{t}=-\gamma u_{t}=-\gamma \xi
$$

namely

$$
\frac{\partial \xi}{\partial v}+\gamma \xi=0, \quad(t, x) \in\left[0, t^{*}\right) \times \partial \Omega
$$

From (3.2), we are led to

$$
\xi(x, 0)=u_{t}(x, 0)=\frac{1}{g^{\prime}}\left(\nabla \cdot\left(\rho\left(\left|\nabla u_{0}\right|^{p}\right) \nabla u_{0}\right)+k(0) a(x) f\left(u_{0}\right)\right) \geq 0, \quad x \in \bar{\Omega}
$$

According to (3.4)-(3.6) and the maximum principle in [25], we can obtain

$$
u_{t}=\xi(x, t) \geq 0, \quad(t, x) \in\left[0, t^{*}\right) \times \bar{\Omega} .
$$

The proof is complete. 
In the sequel, the main result Theorem 3.1 will be stated under Lemma 3.1 and the auxiliary functions defined by (2.1) and (2.2).

Theorem 3.1 Let $u$ be a classical solution of problem (1.1). And assume that (3.1)-(3.2) hold and the functions $\rho, g, f, k, a$ satisfy the following conditions:

$$
\begin{aligned}
& 1 \leq \rho(s) \leq 1+\alpha, \quad g^{\prime \prime}(s) \leq 0, \quad s>0, \\
& \int_{\Omega} a(x) s f(s) \mathrm{d} x \geq 2(1+\alpha) \int_{\Omega} a(x) F(s) \mathrm{d} x, \quad s>0, \\
& \Psi(0):=-\gamma \int_{\partial \Omega} u_{0}^{2} \mathrm{~d} S-\int_{\Omega}\left|\nabla u_{0}\right|^{2} \mathrm{~d} x+2 k(0) \int_{\Omega} a(x) F\left(u_{0}\right) \mathrm{d} x>0,
\end{aligned}
$$

where $u_{0}$ is the initial value and $\alpha$ is a positive constant. Then $u$ blows up at some finite time $T \leq T^{*}$ in the measure $\Phi(t)$. And

$$
T^{*}=\frac{\Phi(0)}{2 \alpha(1+\alpha) \Psi(0)} .
$$

Proof Obviously, (2.6) also holds. Then, from assumptions (3.7) and (3.8), we rewrite (2.6) as

$$
\begin{aligned}
\Phi^{\prime}(t) & \geq-2 \gamma(1+\alpha) \int_{\partial \Omega} u^{2} \mathrm{~d} S-2(1+\alpha) \int_{\Omega}|\nabla u|^{2} \mathrm{~d} x+4(1+\alpha) \int_{\Omega} k(t) a(x) F(u) \mathrm{d} x \\
& =2(1+\alpha) \Psi(t) .
\end{aligned}
$$

Differentiating $\Psi(t)$ in (2.2), we obtain

$$
\begin{aligned}
\Psi^{\prime}(t)= & -2 \gamma \int_{\partial \Omega} u u_{t} \mathrm{~d} S-2 \int_{\Omega} \nabla u \cdot \nabla u_{t} \mathrm{~d} x+2 k^{\prime}(t) \int_{\Omega} a(x) F(u) \mathrm{d} x \\
& +2 k(t) \int_{\Omega} a(x) f(u) u_{t} \mathrm{~d} x \\
\geq & -2 \gamma \int_{\partial \Omega} u u_{t} \mathrm{~d} S-2 \int_{\Omega} \nabla u \cdot \nabla u_{t} \mathrm{~d} x+2 k(t) \int_{\Omega} a(x) f(u) u_{t} \mathrm{~d} x \\
\geq & -2 \gamma \int_{\partial \Omega} \rho\left(|\nabla u|^{p}\right) u u_{t} \mathrm{~d} S-2 \int_{\Omega} \rho\left(|\nabla u|^{p}\right) \nabla u \cdot \nabla u_{t} \mathrm{~d} x \\
& +2 k(t) \int_{\Omega} a(x) f(u) u_{t} \mathrm{~d} x,
\end{aligned}
$$

where (3.2) and (3.7) are used. Moreover, it follows from Gauss's law and Robin's boundary condition that

$$
\begin{aligned}
\Psi^{\prime}(t) \geq & 2 \int_{\partial \Omega} \rho\left(|\nabla u|^{p}\right) u_{t} \frac{\partial u}{\partial v} \mathrm{~d} S-2 \int_{\Omega} \rho\left(|\nabla u|^{p}\right) \nabla u \cdot \nabla u_{t} \mathrm{~d} x \\
& +2 k(t) \int_{\Omega} a(x) f(u) u_{t} \mathrm{~d} x \\
= & 2 \int_{\Omega} \nabla \cdot\left(\rho\left(|\nabla u|^{p}\right) \nabla u u_{t}\right) \mathrm{d} x-2 \int_{\Omega} \rho\left(|\nabla u|^{p}\right) \nabla u \cdot \nabla u_{t} \mathrm{~d} x \\
& +2 k(t) \int_{\Omega} a(x) f(u) u_{t} \mathrm{~d} x
\end{aligned}
$$




$$
\begin{aligned}
& =2 \int_{\Omega} u_{t} \nabla \cdot\left(\rho\left(|\nabla u|^{p}\right) \nabla u\right) \mathrm{d} x+2 k(t) \int_{\Omega} a(x) f(u) u_{t} \mathrm{~d} x \\
& =2 \int_{\Omega} g^{\prime}(u) u_{t}^{2} \mathrm{~d} x \\
& \geq 0 .
\end{aligned}
$$

Therefore, $\Psi(t)$ is a nondecreasing function in $t$. By virtue of (3.9), it is easy to get that

$$
\Psi(t) \geq \Psi(0)>0, \quad \forall t \in(0, T)
$$

Consequently, (3.10) yields

$$
\Phi^{\prime}(t)>0
$$

With the help of (3.10)-(3.11), we obtain

$$
\begin{aligned}
2(1+\alpha) \Psi(t) \Phi^{\prime}(t) & \leq\left(\Phi^{\prime}(t)\right)^{2}=\left(2 \int_{\Omega} u g^{\prime}(u) u_{t} \mathrm{~d} x\right)^{2} \\
& \leq 4 \int_{\Omega} g^{\prime}(u) u^{2} \mathrm{~d} x \int_{\Omega} g^{\prime}(u) u_{t}^{2} \mathrm{~d} x
\end{aligned}
$$

where Schwarz's inequality is applied. Integrating by parts and (3.7), we are led to

$$
\begin{aligned}
G(u) & =2 \int_{0}^{u} y g^{\prime}(y) \mathrm{d} y \\
& =\int_{0}^{u} g^{\prime}(y) \mathrm{d} y^{2} \\
& =g^{\prime}(u) u^{2}-\int_{0}^{u} y^{2} g^{\prime \prime}(y) \mathrm{d} y \\
& \geq g^{\prime}(u) u^{2} .
\end{aligned}
$$

Therefore, (3.13) can be replaced by

$$
(1+\alpha) \Psi(t) \Phi^{\prime}(t) \leq 2 \int_{\Omega} G(u) \mathrm{d} x \int_{\Omega} g^{\prime}(u) u_{t}^{2} \mathrm{~d} x \leq \Phi(t) \Psi^{\prime}(t),
$$

namely

$$
\left(\Psi(t) \Phi(t)^{-(\alpha+1)}\right)^{\prime} \geq 0
$$

We integrate (3.15) from 0 to $t$ and obtain

$$
\Psi(t) \Phi(t)^{-(\alpha+1)} \geq \Psi(0) \Phi(0)^{-(\alpha+1)} .
$$

(3.10) and (3.16) imply

$$
\Phi^{\prime}(t) \Phi(t)^{-(\alpha+1)} \geq 2(1+\alpha) \Psi(0) \Phi(0)^{-(\alpha+1)} .
$$


When $\alpha>0$, integrating (3.17) from 0 to $t$ again, we have

$$
\Phi(t)^{-\alpha} \leq-2 \alpha(1+\alpha) \Psi(0) \Phi(0)^{-(\alpha+1)} t+\Phi(0)^{-\alpha} .
$$

Hence, it follows from (3.18) that the solution $u$ of (1.1) blows up at some finite time $T<T^{*}$ in the measure $\Phi(t)$ and

$$
T<T^{*}=\frac{\Phi(0)}{2 \alpha(1+\alpha) \Psi(0)} .
$$

\section{A lower bound for blow-up time}

In this subsection, a lower bound for the blow-up time $t^{*}$ is given. Now we will make the following assumptions on the functions $k, \rho, a, f, g$ :

$$
\begin{aligned}
& a(x) f(s) \leq c_{1}+c_{2} s^{\beta}, \quad s>0, \\
& \rho(s) \geq b_{1}+b_{2} s^{2 q}, \quad g^{\prime}(s) \geq m_{0}, \quad s>0, \\
& \frac{k^{\prime}(t)}{k(t)} \leq \eta, \quad k(t) \leq \eta_{0}, \quad t>0,
\end{aligned}
$$

where $c_{1}, q$ are nonnegative constants, $b_{1}, b_{2}, c_{2}, \eta, m_{0}, \beta, \eta_{0}$ are some positive constants, and they satisfy $\beta>2 p q+1$. Now we define the new auxiliary functions to prove the main result in this section:

$$
A(t)=k^{\delta_{1}}(t) \int_{\Omega} B(u) \mathrm{d} x, \quad t \geq 0, \quad B(s)=\delta_{2} \int_{0}^{s} g^{\prime}(y) y^{\delta_{2}-1} \mathrm{~d} y, \quad s \geq 0,
$$

where

$$
\begin{aligned}
& \delta_{1}=\frac{2 r(p q+1)+p q(n-2)}{\beta-1}, \quad \delta_{2}=2 r(p q+1)-2 p q, \\
& \mu \geq r=\max \left\{\frac{(n-2)(\beta-2 p q-1)}{4(p q+1)}, 1\right\} .
\end{aligned}
$$

The following is our main result.

Theorem 4.1 Let $u$ be a classical solution of problem (1.1). Assume that conditions (4.1)(4.3) on $k, \rho, a, f, g$ hold. And $u$ becomes unbounded in the measure $A(t)$ at some finite time $t^{*}$. Then the blow-up time $t^{*}$ is bounded and it satisfies

$$
t^{*} \geq \int_{A(0)}^{\infty} \frac{1}{\delta_{1} \eta \tau+C_{1} \tau^{\frac{\delta_{2}-1}{\delta_{2}}}+C_{2} \tau^{\frac{4 r(p q+1)+2 p q(n-2)-(n-2)(\beta-1)}{4 r(p q+1)+2 p q(n-2)-n(\beta-1)}}} d \tau .
$$

Here

$$
\begin{aligned}
& C_{1}=\frac{\eta_{0}^{\frac{\delta_{1}+\delta_{2}}{\delta_{2}}}|\Omega|^{\frac{1}{\delta_{2}}} c_{1} \delta_{2}}{m_{0}^{\frac{\delta_{2}-1}{\delta_{2}}}} \\
& C_{2}=\frac{4 r(p q+1)+2 p q(n-2)-n(\beta-1)}{4 r(p q+1)+2 p q(n-2)} c_{2} \delta_{2}\left(C_{0}^{2}\left(1+\frac{1}{M}\right)\right)^{\frac{n(\beta-1)}{4 r(p q+1)+2 p q(n-2)}}
\end{aligned}
$$




$$
\times \varepsilon^{-\frac{n(\beta-1)}{4 r(p q+1)+2 p q(n-2)}}\left(\frac{1}{m_{0}}\right)^{\frac{4 r(p q+1)+2 p q(n-2)-(n-2)(\beta-1)}{4 r(p q+1)+2 p q(n-2)-n(\beta-1)}}
$$

where $C_{0}$ is a Sobolev embedding constant depending on $n$ and $\Omega,|\Omega|$ stands for the volume of $\Omega$.

$$
\begin{aligned}
& \varepsilon=\frac{b_{2} \delta_{2}\left(\delta_{2}-1\right) M^{p q}(4 r(p q+1)+2 p q(n-2))}{n(\beta-1)(r(p q+1))^{2(p q+1)} c_{2} \delta_{2}\left(C_{0}^{2}\left(1+\frac{1}{M}\right)\right)^{\frac{n(\beta-1)}{4 r(p q+1)+2 p q(n-2)}}}, \\
& M=\frac{\lambda-r \gamma(p q+1)\left(\frac{n}{L_{0}}+\left(\frac{d}{L_{0}}\right)^{2} \theta_{0}\right)}{1+r \gamma(p q+1) \theta_{0}^{-1}}, \quad \theta_{0}=\frac{\lambda L_{0}^{2}-r \gamma(p q+1) n L_{0}}{2 \gamma(p q+1) d^{2}} .
\end{aligned}
$$

Proof From Gauss's law and conditions (4.1) - -(4.3), we obtain

$$
\begin{aligned}
& A^{\prime}(t)=\delta_{1} k^{\delta_{1}-1}(t) k^{\prime}(t) \int_{\Omega} B(u) \mathrm{d} x+k^{\delta_{1}}(t) \int_{\Omega} B^{\prime}(u) u_{t} \mathrm{~d} x \\
& =\delta_{1} k^{\delta_{1}}(t) \frac{k^{\prime}(t)}{k(t)} \int_{\Omega} B(u) \mathrm{d} x+\delta_{2} k^{\delta_{1}}(t) \int_{\Omega} u^{\delta_{2}-1} g^{\prime}(u) u_{t} \mathrm{~d} x \\
& \leq \delta_{1} \eta A(t)+\delta_{2} k^{\delta_{1}}(t) \int_{\Omega} u^{\delta_{2}-1}\left(\nabla \cdot\left(\rho\left(|\nabla u|^{p}\right) \nabla u\right)+a(x) k(t) f(u)\right) \mathrm{d} x \\
& =\delta_{1} \eta A(t)+\delta_{2} k^{\delta_{1}}(t) \int_{\Omega} \nabla \cdot\left(u^{\delta_{2}-1} \rho\left(|\nabla u|^{p}\right) \nabla u\right) \mathrm{d} x-\delta_{2}\left(\delta_{2}-1\right) k^{\delta_{1}}(t) \\
& \times \int_{\Omega} u^{\delta_{2}-2} \rho\left(|\nabla u|^{p}\right)|\nabla u|^{2} \mathrm{~d} x+\delta_{2} k^{\delta_{1}+1}(t) \int_{\Omega} u^{\delta_{2}-1} a(x) f(u) \mathrm{d} x \\
& =\delta_{1} \eta A(t)+\delta_{2} k^{\delta_{1}}(t) \int_{\partial \Omega} u^{\delta_{2}-1} \rho\left(|\nabla u|^{p}\right) \frac{\partial u}{\partial v} \mathrm{~d} S-\delta_{2}\left(\delta_{2}-1\right) k^{\delta_{1}}(t) \\
& \times \int_{\Omega} u^{\delta_{2}-2} \rho\left(|\nabla u|^{p}\right)|\nabla u|^{2} \mathrm{~d} x+\delta_{2} k^{\delta_{1}+1}(t) \int_{\Omega} u^{\delta_{2}-1} a(x) f(u) \mathrm{d} x \\
& \leq \delta_{1} \eta A(t)-\gamma \delta_{2} k^{\delta_{1}}(t) \int_{\partial \Omega} u^{\delta_{2}} \rho\left(|\nabla u|^{p}\right) \mathrm{d} S-\delta_{2}\left(\delta_{2}-1\right) k^{\delta_{1}}(t) \\
& \times \int_{\Omega} u^{\delta_{2}-2}\left(b_{1}+b_{2}|\nabla u|^{2 p q}\right)|\nabla u|^{2} \mathrm{~d} x+\delta_{2} k^{\delta_{1}+1}(t) \int_{\Omega} u^{\delta_{2}-1}\left(c_{1}+c_{2} u^{\beta}\right) \mathrm{d} x \\
& \leq \delta_{1} \eta A(t)-\delta_{2} b_{2}\left(\delta_{2}-1\right) k^{\delta_{1}}(t) \int_{\Omega} u^{\delta_{2}-2}|\nabla u|^{2 p q+2} \mathrm{~d} x+c_{1} \delta_{2} k^{\delta_{1}+1}(t) \\
& \times \int_{\Omega} u^{\delta_{2}-1} \mathrm{~d} x+c_{2} \delta_{2} k^{\delta_{1}+1}(t) \int_{\Omega} u^{\delta_{2}+\beta-1} \mathrm{~d} x .
\end{aligned}
$$

Then we replace $u^{r}$ with $v$ in (4.11) and have

$$
\begin{aligned}
A^{\prime}(t) \leq & \delta_{1} \eta A(t)-\frac{\delta_{2} b_{2}\left(\delta_{2}-1\right)}{r^{2 p q+2}} k^{\delta_{1}}(t) \int_{\Omega}|\nabla v|^{2 p q+2} \mathrm{~d} x+c_{1} \delta_{2} k^{\delta_{1}+1}(t) \int_{\Omega} v^{\frac{\delta_{2}-1}{r}} \mathrm{~d} x \\
& +c_{2} \delta_{2} k^{\delta_{1}+1}(t) \int_{\Omega} v^{2(p q+1)+\frac{\beta-2 p q-1}{r}} \mathrm{~d} x .
\end{aligned}
$$

Similar to the proof of inequality (2.12), we can derive

$$
\int_{\Omega}\left|\nabla v^{p q+1}\right|^{2} \mathrm{~d} x \geq M \int_{\Omega} v^{2 p q+2} \mathrm{~d} x
$$


where $M>0$ is defined by (4.10). It follows from Hölder's inequality and (4.13) that

$$
\begin{aligned}
\int_{\Omega}\left|\nabla v^{p q+1}\right|^{2} \mathrm{~d} x & =(p q+1)^{2} \int_{\Omega} v^{2 p q}|\nabla v|^{2} \mathrm{~d} x \\
& \leq(p q+1)^{2}\left(\int_{\Omega} v^{2 p q+2} \mathrm{~d} x\right)^{\frac{p q}{p q+1}}\left(\int_{\Omega}|\nabla v|^{2 p q+2} \mathrm{~d} x\right)^{\frac{1}{p q+1}} \\
& \leq \frac{(p q+1)^{2}}{M^{\frac{p q}{p q+1}}}\left(\int_{\Omega}\left|\nabla \nu^{p q+1}\right|^{2} \mathrm{~d} x\right)^{\frac{p q}{p q+1}}\left(\int_{\Omega}|\nabla v|^{2 p q+2} \mathrm{~d} x\right)^{\frac{1}{p q+1}} .
\end{aligned}
$$

That is,

$$
\int_{\Omega}|\nabla v|^{2 p q+2} \mathrm{~d} x \geq \frac{M^{p q}}{(p q+1)^{2 p q+2}} \int_{\Omega}\left|\nabla v^{p q+1}\right|^{2} \mathrm{~d} x .
$$

Therefore, (4.12) can be rewritten as

$$
\begin{aligned}
A^{\prime}(t) \leq & \delta_{1} \eta A(t)-\frac{\delta_{2} b_{2}\left(\delta_{2}-1\right) M^{p q}}{(r(p q+1))^{2 p q+2}} k^{\delta_{1}}(t) \int_{\Omega}\left|\nabla v^{p q+1}\right|^{2} \mathrm{~d} x+c_{1} \delta_{2} k^{\delta_{1}+1}(t) \\
& \times \int_{\Omega} v^{\frac{\delta_{2}-1}{r}} \mathrm{~d} x+c_{2} \delta_{2} k^{\delta_{1}+1}(t) \int_{\Omega} v^{2(p q+1)+\frac{\beta-2 p q-1}{r}} \mathrm{~d} x .
\end{aligned}
$$

In what follows, we estimate the last two terms on the right-hand side of (4.16). Hölder's inequality and (4.3) yield

$$
\begin{aligned}
k^{\delta_{1}+1}(t) \int_{\Omega} v^{\frac{\delta_{2}-1}{r}} \mathrm{~d} x & \leq\left(\int_{\Omega} k^{\delta_{1}}(t) v^{\frac{\delta_{2}}{r}} \mathrm{~d} x\right)^{\frac{\delta_{2}-1}{\delta_{2}}} k^{\frac{\delta_{1}+\delta_{2}}{\delta_{2}}}(t)|\Omega|^{\frac{1}{\delta_{2}}} \\
\leq & \left(\int_{\Omega} k^{\delta_{1}}(t) v^{\frac{\delta_{2}}{r}} \mathrm{~d} x\right)^{\frac{\delta_{2}-1}{\delta_{2}}} \eta_{0}^{\frac{\delta_{1}+\delta_{2}}{\delta_{2}}}|\Omega|^{\frac{1}{\delta_{2}}} \\
k^{\delta_{1}+1}(t) \int_{\Omega} v^{2(p q+1)+\frac{\beta-2 p q-1}{r}} \mathrm{~d} x \leq & \left(k^{\delta_{1}}(t) \int_{\Omega} v^{\frac{\delta_{2}}{r}} \mathrm{~d} x\right)^{\frac{4 r(p q+1)-(n-2)(\beta-2 p q-1)}{4 r(p q+1)+2 p q(n-2)}} \\
& \times\left(k^{\frac{n \delta_{1}}{n-2}}(t) \int_{\Omega} v^{\frac{2 n(p q+1)}{n-2}} \mathrm{~d} x\right)^{\frac{(n-2)(\beta-1)}{4 r(p q+1)+2 p q(n-2)}} .
\end{aligned}
$$

Now we deal with (4.18). Using Sobolev's inequality and (4.13), we get

$$
\begin{aligned}
\left(\int_{\Omega} v^{\frac{2 n(p q+1)}{n-2}} \mathrm{~d} x\right)^{\frac{n-2}{2 n}} & \leq C_{0}\left(\int_{\Omega} v^{2(p q+1)} \mathrm{d} x+\int_{\Omega}\left|\nabla v^{p q+1}\right|^{2} \mathrm{~d} x\right)^{\frac{1}{2}} \\
& \leq C_{0}\left(\int_{\Omega} \frac{1}{M}\left|\nabla \nu^{p q+1}\right|^{2} \mathrm{~d} x+\int_{\Omega}\left|\nabla \nu^{p q+1}\right|^{2} \mathrm{~d} x\right)^{\frac{1}{2}} \\
& \leq C_{0}\left(1+\frac{1}{M}\right)^{\frac{1}{2}}\left(\int_{\Omega}\left|\nabla \nu^{p q+1}\right|^{2} \mathrm{~d} x\right)^{\frac{1}{2}} .
\end{aligned}
$$

Namely,

$$
\int_{\Omega} v^{\frac{2 n(p q+1)}{n-2}} \mathrm{~d} x \leq C_{0}^{\frac{2 n}{n-2}}\left(1+\frac{1}{M}\right)^{\frac{n}{n-2}}\left(\int_{\Omega}\left|\nabla \nu^{p q+1}\right|^{2} \mathrm{~d} x\right)^{\frac{n}{n-2}} .
$$


The substitution of (4.19) into (4.18) and Young's inequality imply

$$
\begin{aligned}
& k^{\delta_{1}+1}(t) \int_{\Omega} v^{2(p q+1)+\frac{\beta-2 p q-1}{r}} \mathrm{~d} x \\
& \leq\left(k^{\delta_{1}}(t) \int_{\Omega} v^{\frac{\delta_{2}}{r}} \mathrm{~d} x\right)^{\frac{4 r(p q+1)-(n-2)(\beta-2 p q-1)}{4 r(p q+1)+2 p q(n-2)}}\left\{k^{\frac{n \delta_{1}}{n-2}}(t) C_{0}^{\frac{2 n}{n-2}}\left(1+\frac{1}{M}\right)^{\frac{n}{n-2}}\right. \\
& \left.\times\left(\int_{\Omega}\left|\nabla v^{p q+1}\right|^{2} \mathrm{~d} x\right)^{\frac{n}{n-2}}\right\}^{\frac{(n-2)(\beta-1)}{4 r(p q+1)+2 p q(n-2)}} \\
& =\left\{\left(k^{\delta_{1}}(t) \int_{\Omega} v^{\frac{\delta_{2}}{r}} \mathrm{~d} x\right)^{\frac{4 r(p q+1)+2 p q(n-2)-(n-2)(\beta-1)}{4 r(p q+1)+2 p q(n-2)-n(\beta-1)}}\right\}^{\frac{4 r(p q+1)+2 p q(n-2)-n(\beta-1)}{4 r(p q+1)+2 p q(n-2)}} \\
& \times\left\{C_{0}^{2}\left(1+\frac{1}{M}\right)\right\}^{\frac{n(\beta-1)}{4 r(p q+1)+2 p q(n-2)}} \cdot\left(k^{\delta_{1}}(t) \int_{\Omega}\left|\nabla v^{p q+1}\right|^{2} \mathrm{~d} x\right)^{\frac{n(\beta-1)}{4 r(p q+1)+2 p q(n-2)}} \\
& =\left\{C_{0}^{2}\left(1+\frac{1}{M}\right)\right\}^{\frac{n(\beta-1)}{4 r(p q+1)+2 p q(n-2)}} \cdot\left\{\varepsilon^{-\frac{n(\beta-1)}{4 r(p q+1)+2 p q(n-2)-n(\beta-1)}}\right. \\
& \left.\times\left(k^{\delta_{1}}(t) \int_{\Omega} v^{\frac{\delta_{2}}{r}} \mathrm{~d} x\right)^{\frac{4 r(p q+1)+2 p q(n-2)-(n-2)(\beta-1)}{4 r(p q+1)+2 p q(n-2)-n(\beta-1)}}\right\}^{\frac{4 r(p q+1)+2 p q(n-2)-n(\beta-1)}{4 r(p q+1)+2 p q(n-2)}} \\
& \times\left(\varepsilon k^{\delta_{1}}(t) \int_{\Omega}\left|\nabla v^{p q+1}\right|^{2} \mathrm{~d} x\right)^{\frac{n(\beta-1)}{4 r(p q+1)+2 p q(n-2)}} \\
& \leq\left\{C_{0}^{2}\left(1+\frac{1}{M}\right)\right\}^{\frac{n(\beta-1)}{4 r(p q+1)+2 p q(n-2)}} \cdot\left\{\frac{4 r(p q+1)+2 p q(n-2)-n(\beta-1)}{4 r(p q+1)+2 p q(n-2)}\right. \\
& \times \varepsilon^{-\frac{n(\beta-1)}{4 r(p q+1)+2 p q(n-2)}} \cdot\left(k^{\delta_{1}}(t) \int_{\Omega} v^{\frac{\delta_{2}}{r}} \mathrm{~d} x\right)^{\frac{4 r(p q+1)+2 p q(n-2)-(n-2)(\beta-1)}{4 r(p q+1)+2 p q(n-2)-n(\beta-1)}} \\
& \left.+\frac{n(\beta-1)}{4 r(p q+1)+2 p q(n-2)} \cdot\left(\varepsilon k^{\delta_{1}}(t) \int_{\Omega}\left|\nabla v^{p q+1}\right|^{2} \mathrm{~d} x\right)\right\} .
\end{aligned}
$$

We insert (4.17)-(4.20) into (4.16) and obtain

$$
\begin{aligned}
& A^{\prime}(t) \leq \delta_{1} \eta A(t)-\frac{\delta_{2} b_{2}\left(\delta_{2}-1\right) M^{p q}}{(r(p q+1))^{2 p q+2}} k^{\delta_{1}}(t) \int_{\Omega}\left|\nabla \nu^{p q+1}\right|^{2} \mathrm{~d} x+\eta_{0}^{\frac{\delta_{1}+\delta_{2}}{\delta_{2}}}|\Omega|^{\frac{1}{\delta_{2}}} \\
& \times c_{1} \delta_{2}\left(k^{\delta_{1}}(t) \int_{\Omega} v^{\frac{\delta_{2}}{r}} \mathrm{~d} x\right)^{\frac{\delta_{2}-1}{\delta_{2}}}+c_{2} \delta_{2}\left\{C_{0}^{2}\left(1+\frac{1}{M}\right)\right\}^{\frac{n(\beta-1)}{4 r(p q+1)+2 p q(n-2)}} \\
& \times\left\{\frac{4 r(p q+1)+2 p q(n-2)-n(\beta-1)}{4 r(p q+1)+2 p q(n-2)} \varepsilon^{-\frac{n(\beta-1)}{4 r(p q+1)+2 p q(n-2)}}\right. \\
& \times\left(k^{\delta_{1}}(t) \int_{\Omega} v^{\frac{\delta_{2}}{r}} \mathrm{~d} x\right)^{\frac{4 r(p q+1)+2 p q(n-2)-(n-2)(\beta-1)}{4 r(p q+1)+2 p q(n-2)-n(\beta-1)}}+\frac{n(\beta-1)}{4 r(p q+1)+2 p q(n-2)} \\
& \left.\times\left(\varepsilon k^{\delta_{1}}(t) \int_{\Omega}\left|\nabla \nu^{p q+1}\right|^{2} \mathrm{~d} x\right)\right\} .
\end{aligned}
$$

From the definition of $\varepsilon$, the terms containing $\int_{\Omega}\left|\nabla v^{p q+1}\right|^{2} \mathrm{~d} x$ in (4.21) are offset, that is,

$$
A^{\prime}(t) \leq \delta_{1} \eta A(t)+\eta_{0}^{\frac{\delta_{1}+\delta_{2}}{\delta_{2}}}|\Omega|^{\frac{1}{\delta_{2}}} c_{1} \delta_{2}\left(k^{\delta_{1}}(t) \int_{\Omega} v^{\frac{\delta_{2}}{r}} \mathrm{~d} x\right)^{\frac{\delta_{2}-1}{\delta_{2}}}+c_{2} \delta_{2} \varepsilon^{-\frac{n(\beta-1)}{4 r(p q+1)+2 p q(n-2)}}
$$




$$
\begin{aligned}
& \times \frac{4 r(p q+1)+2 p q(n-2)-n(\beta-1)}{4 r(p q+1)+2 p q(n-2)}\left(C_{0}^{2}\left(1+\frac{1}{M}\right)\right)^{\frac{n(\beta-1)}{4 r(p q+1)+2 p q(n-2)}} \\
& \times\left(k^{\delta_{1}}(t) \int_{\Omega} v^{\frac{\delta_{2}}{r}} \mathrm{~d} x\right)^{\frac{4 r(p q+1)+2 p q(n-2)-(n-2)(\beta-1)}{4 r(p q+1)+2 p q(n-2)-n(\beta-1)}}
\end{aligned}
$$

By $g^{\prime}(s)>0$ in (4.2), we get

$$
B(u)=\delta_{2} \int_{0}^{u} g^{\prime}(y) y^{\delta_{2}-1} \mathrm{~d} y \geq m_{0} \delta_{2} \int_{0}^{u} y^{\delta_{2}-1} \mathrm{~d} y=m_{0} u^{\delta_{2}} .
$$

That is,

$$
v^{\frac{\delta_{2}}{r}}=u^{\delta_{2}} \leq \frac{1}{m_{0}} B(u)
$$

Then

$$
A^{\prime}(t) \leq \delta_{1} \eta A(t)+C_{1} A(t)^{\frac{\delta_{2}-1}{\delta_{2}}}+C_{2} A(t)^{\frac{4 r(p q+1)+2 p q(n-2)-(n-2)(\beta-1)}{4 r(p q+1)+2 p q(n-2)-n(\beta-1)}}
$$

where $C_{1}, C_{2}$ are defined as (4.7) and (4.8). Integrating (4.22) from 0 to $t$, we have

$$
\int_{A(0)}^{A(t)} \frac{\mathrm{d} \tau}{\delta_{1} \eta \tau+C_{1} \tau^{\frac{\delta_{2}-1}{\delta_{2}}}+C_{2} \tau^{\frac{4 r(p q+1)+2 p q(n-2)-(n-2)(\beta-1)}{4 r(p q+1)+2 p q(n-2)-n(\beta-1)}}} \leq t
$$

We take the limit $t \rightarrow t^{*-}$ to get

$$
t^{*} \geq \int_{A(0)}^{\infty} \frac{d \tau}{\delta_{1} \eta \tau+C_{1} \tau^{\frac{\delta_{2}-1}{\delta_{2}}}+C_{2} \tau^{\frac{4 r(p q+1)+2 p q(n-2)-(n-2)(\beta-1)}{4 r(p q+1)+2 p q(n-2)-n(\beta-1)}}}
$$

Thus, if $u$ blows up in the measure $A(t)$ at some finite time $t^{*}$, and there is a lower bound for blow-up time.

\section{Applications}

As applications, the section shows that two examples illustrate our main results.

Example 5.1 Assume that $u$ is a classical solution of the following equation:

$$
\left\{\begin{array}{l}
(3 u-\ln (1+u))_{t}=\nabla \cdot\left(\left(2+|\nabla u|^{\frac{5}{4}}\right) \nabla u\right)+e^{3 t}\left(2-\frac{|x|^{2}}{4}\right) u^{2}\left(\int_{\Omega} u \mathrm{~d} x\right)^{\frac{1}{6}} \\
\quad(x, t) \in \Omega \times(0, T) \\
\frac{\partial u}{\partial v}+\frac{1}{3} u=0, \quad(x, t) \in \partial \Omega \times(0, T) \\
u(x, 0)=u_{0}(x)=4-\frac{|x|^{2}}{4}, \quad x \in \bar{\Omega}
\end{array}\right.
$$

where $\Omega=\left\{x=\left(x_{1}, x_{2}, x_{3}\right) \mid x_{1}^{2}+x_{2}^{2}+x_{3}^{2}<4\right\}$. Then $u$ cannot blow up in the measure $\Phi(t)$, where

$$
\Phi(t)=\int_{\Omega}-2 u+3 u^{2}+\ln (1+u) \mathrm{d} x
$$


Proof Compared with problem (1.1), it is obvious that

$$
\begin{aligned}
& g(u)=3 u-\ln (1+u), \quad \rho(s)=2+s^{\frac{1}{4}}, \quad f(u)=u^{2}\left(\int_{\Omega} u \mathrm{~d} x\right)^{\frac{1}{6}}, \\
& k(t)=e^{3 t}, \quad a(x)=2-\frac{|x|^{2}}{4}, \quad u_{0}(x)=4-\frac{|x|^{2}}{4}, \quad p=5 .
\end{aligned}
$$

Set $q=\frac{1}{8}, a_{1}=\kappa=2, l=1, \gamma=\frac{1}{3}, c=3, m=\frac{1}{6}, \lambda=2.4547$. And they satisfy the conditions of Theorem 2.1. Hence, we get that $u$ cannot blow up in the measure $\Phi(t)$.

Example 5.2 Suppose that $u$ is a classical solution of the following equation:

$$
\left\{\begin{array}{l}
(3 u+\ln (1+u))_{t}=\nabla \cdot\left(\left(5+\frac{1}{3+|\nabla u|^{3}}\right) \nabla u\right)+\left(3-2 e^{-t}\right)\left(2-\frac{|x|^{2}}{4}\right) u^{2}, \\
\quad(x, t) \in \Omega \times(0, T), \\
\frac{\partial u}{\partial v}+\frac{1}{3} u=0, \quad(x, t) \in \partial \Omega \times(0, T), \\
u(x, 0)=u_{0}(x)=2-\frac{|x|^{2}}{8}, \quad x \in \bar{\Omega},
\end{array}\right.
$$

where $\Omega=\left\{x=\left(x_{1}, x_{2}, x_{3}\right) \mid x_{1}^{2}+x_{2}^{2}+x_{3}^{2}<4\right\}$. Then $u$ will blow up at $t^{*}<0.0552$ in the measure $\Phi(t)$, where

$$
\Phi(t)=\int_{\Omega} G(u) \mathrm{d} x=\int_{\Omega}\left(3 u^{2}+2 u-2 \ln (1+u)\right) \mathrm{d} x .
$$

Moreover, the blow-up time $t^{*}$ is also bounded below and

$$
t^{*} \geq 0.0004
$$

Proof Compared with (1.1), we have

$$
\begin{aligned}
& g(u)=3 u+\ln (1+u), \quad \rho\left(|\nabla u|^{3}\right)=5+\frac{1}{3+|\nabla u|^{3}}, \quad f(u)=u^{2}, \\
& k(t)=3-2 e^{-t}, \quad a(x)=2-\frac{|x|^{2}}{4}, \quad u_{0}(x)=2-\frac{|x|^{2}}{8}, \quad p=3, \\
& G(u)=2 \int_{0}^{u} y g^{\prime}(y) \mathrm{d} y=3 u^{2}+2 u-2 \ln (1+u), \\
& F(u)=\int_{0}^{u} y^{2} \mathrm{~d} y=\frac{1}{3} u^{3} .
\end{aligned}
$$

Firstly, we demonstrate that $u$ blows up in finite time and obtain an upper bound for blowup time. Define following functions:

$$
\begin{aligned}
& \Phi(t)=\int_{\Omega} G(u) \mathrm{d} x=\int_{\Omega}\left(3 u^{2}+2 u-2 \ln (1+u)\right) \mathrm{d} x \\
& \Psi(t)=-\frac{1}{3} \int_{\partial \Omega} u^{2} \mathrm{~d} S-\int_{\Omega}|\nabla u|^{2} \mathrm{~d} x+2\left(3-2 e^{-t}\right) \int_{\Omega}\left(2-\frac{|x|^{2}}{4}\right) F(u) \mathrm{d} x .
\end{aligned}
$$


Set $\alpha=\frac{13}{3}$. We verify that conditions (3.2), (3.7), and (3.8) are satisfied. We calculate that

$$
\begin{aligned}
\Phi(0)= & \int_{\Omega} G\left(u_{0}(x)\right) \mathrm{d} x=\int_{\Omega}\left(3 u_{0}^{2}+2 u_{0}-2 \ln \left(1+u_{0}\right)\right) \mathrm{d} x \\
= & \int_{\Omega}\left(3\left(2-\frac{|x|^{2}}{8}\right)^{2}+2\left(2-\frac{|x|^{2}}{8}\right)-2 \ln \left(3-\frac{|x|^{2}}{8}\right)\right) \mathrm{d} x \\
= & \int_{0}^{2 \pi} \mathrm{d} \theta \int_{0}^{\pi} \mathrm{d} \varphi \int_{0}^{2}\left(3\left(2-\frac{r^{2}}{8}\right)^{2}+2\left(2-\frac{r^{2}}{8}\right)-2 \ln \left(3-\frac{r^{2}}{8}\right)\right) r^{2} \sin \varphi \mathrm{d} r \\
= & 339.703, \\
\Psi(0)= & -\frac{1}{3} \int_{\partial \Omega} u_{0}^{2} \mathrm{~d} S-\int_{\Omega}\left|\nabla u_{0}\right|^{2} \mathrm{~d} x+\frac{2}{3} \int_{\Omega}\left(2-\frac{|x|^{2}}{4}\right) u_{0}^{3} \mathrm{~d} x \\
= & -8 \pi-\int_{0}^{2 \pi} \mathrm{d} \theta \int_{0}^{\pi} \mathrm{d} \varphi \int_{0}^{2} \frac{1}{16} r^{4} \sin \varphi \mathrm{d} r \\
& +\int_{0}^{2 \pi} \mathrm{d} \theta \int_{0}^{\pi} \mathrm{d} \varphi \int_{0}^{2} \frac{2}{3}\left(2-\frac{r^{2}}{4}\right)\left(2-\frac{r^{2}}{8}\right)^{3} r^{2} \sin \varphi \mathrm{d} r \\
= & 133.101 \\
> & 0 .
\end{aligned}
$$

Applying Theorem 3.1, we can obtain $u$ will blow up at $t^{*}<T$ in the measure $\Phi(t)$. And

$$
T=\frac{\Phi(0)}{2 \alpha(1+\alpha) \Psi(0)}=0.0552,
$$

which is an upper bound for the blow-up time.

Now, we will show a lower bound for the blow-up time by applying Theorem 4.1. We choose $c_{1}=0, c_{2}=2, b_{1}=4, b_{2}=\frac{1}{2}, \beta=\eta=2, q=0, m_{0}=\eta_{0}=3$. Obviously, they satisfy (4.1)-(4.3). Let $\delta_{1}=\delta_{2}=2, r=1$. And we have

$$
\begin{aligned}
& B(s)=2 \int_{0}^{s} g^{\prime}(y) y \mathrm{~d} y=3 s^{2}+2 s-2 \ln (1+s), \quad s>0, \\
& A(t)=\left(3-2 e^{-t}\right)^{2} \int_{\Omega}\left(3 u^{2}+2 u-2 \ln (1+u)\right) \mathrm{d} x .
\end{aligned}
$$

Then

$$
A(0)=\int_{\Omega}\left(3 u_{0}^{2}+2 u_{0}-2 \ln \left(1+u_{0}\right)\right) \mathrm{d} x=339.703
$$

We can compute $\lambda=2.4547, L_{0}=d=2,|\Omega|=\frac{32 \pi}{3}$. From [19], we get the embedding constant $C_{0}=4^{\frac{1}{3}} 3^{-\frac{1}{2}} \pi^{-\frac{2}{3}}$. (4.7)-(4.10) imply that $\theta_{0}=2.932, M=2.6327, \varepsilon=12.9082, C_{1}=$ $0, C_{2}=0.01137$. Obviously, we can conclude that $u$ blows up at a finite time $t^{*}$ and $u$ is unbounded in the measure $A(t)$ at a finite time $t^{*}$. By Theorem 4.1, the blow-up time $t^{*}$ is bounded below and

$$
t^{*} \geq \int_{A(0)}^{\infty} \frac{\mathrm{d} \tau}{\varphi(\tau)}=\int_{339.703}^{\infty} \frac{\mathrm{d} \tau}{4 \tau+0.01137 \tau^{3}}=0.0004
$$


Therefore

$$
0.0004 \leq t^{*} \leq 0.0552
$$

\section{Conclusion}

In this paper, we discussed global and blow-up solutions for a class of nonlinear reaction diffusion equations with Robin boundary conditions. By auxiliary functions and a firstorder differential inequality technique, conditions on the data to prove the existence of global solution are established. And applying maximum principles, we obtain the sufficient conditions that guarantee the occurrence of the blow-up. Moreover, an upper bound and a lower bound on blow-up time are discussed.

\section{Acknowledgements}

We are thankful to the editor and the anonymous reviewers for many valuable suggestions to improve this paper.

\section{Funding}

This paper is supported by opening project of State Key Laboratory of Explosion Science and Technology (Beijing Institute of Technology). The opening project number is KFJJ19-06M. This paper is also supported by Key R\&D Program of Shanxi Province (International Cooperation, 201903D421042)

\section{Availability of data and materials}

Data sharing not applicable to this article as no datasets were generated or analysed during the current study.

\section{Competing interests}

The authors declare that they have no competing interests.

\section{Authors' contributions}

HT participated in the design of the study and drafted the manuscript. LZ carried out the theoretical studies and helped to draft the manuscript. All authors read and approved the final manuscript.

\section{Publisher's Note}

Springer Nature remains neutral with regard to jurisdictional claims in published maps and institutional affiliations.

Received: 8 January 2020 Accepted: 18 March 2020 Published online: 31 March 2020

\section{References}

1. Payne, L.E., Philippin, G.A., Vernier Piro, S.: Blow-up phenomena for a semilinear heat equation with nonlinear boundary condition. Nonlinear Anal., Theory Methods Appl. 73, 971-978 (2010)

2. Zhang, L.L.: Blow-up of solutions for a class of nonlinear parabolic equations. Z. Anal. Anwend. 25, 479-486 (2006)

3. Shen, Y., Fang, Z.B.: Bounds for the blow-up time of a porous medium equation with weighted nonlocal source and inner absorption terms. Bound. Value Probl. 2018, 1 (2018)

4. Viglialoro, G.: On the blow-up of parabolic system with damping terms. C. R. Acad. Bulg. Sci. 67, 1223-1232 (2014)

5. Caffarrelli, L.A., Friedman, A.: Blow-up of solutions of nonlinear heat equations. J. Math. Anal. Appl. 129, 409-419 (1988)

6. Enache, C.: Blow-up, global existence and exponential decay estimates for a class of quasilinear parabolic problems. Nonlinear Anal. 69, 2864-2874 (2008)

7. Zhang, L.L., Zhang, N., Li, L.X.: Blow-up solutions and global existence for a kind of quasilinear reaction-diffusion equations. Z. Anal. Anwend. 33, 247-258 (2014)

8. Deng, K., Levine, H.A.: The role of critical exponents in blow-up theorems: the sequel. J. Math. Anal. Appl. 243, 85-126 (2000)

9. Straughan, B.: Explosive Instabilities in Mechanics. Springer, Berlin (1998)

10. Bandle, C., Brunner, H.: Blow-up in diffusion equations: a survey. J. Comput. Appl. Math. 97(1-2), 3-22 (1998)

11. Ding, J.T., Hu, H.J.: Blow-up and global solutions for a class of nonlinear reaction diffusion equations under Dirichlet boundary conditions. J. Math. Anal. Appl. 433, 1718-1735 (2016)

12. Payne, L.E., Schaefer, P.W.: Lower bounds for blow-up time in parabolic problems under Dirichlet conditions. J. Math. Anal. Appl. 328, 1196-1205 (2007)

13. Ding, J.T., Hu, H.J.: Blow-up solutions for nonlinear reaction diffusion equations under Neumann boundary conditions. Appl. Anal. 96, 549-562 (2016)

14. Payne, L.E., Schaefer, P.W.: Lower bounds for blow-up time in parabolic problems under Neumann conditions. Appl. Anal. 85, 1301-1311 (2006)

15. Li, F.S., Li, J.L.: Global existence and blow-up phenomena for p-Laplacian heat equation with inhomogeneous Neumann boundary conditions. Bound. Value Probl. 2014, 219 (2014)

16. LV, X.S., Song, X.F:: Bounds of the blow-up time in parabolic equations with weighted source under nonhomogeneous Neumann boundary condition. Math. Methods Appl. Sci. 37, 1019-1028 (2014) 
17. Marras, M., Vernier Piro, S.: Reaction-diffusion problems under non-local boundary conditions with blow-up solutions. J. Inequal. Appl. 2017, 167 (2017)

18. Ding, J.T., Kou, W.: Blow-up solutions for reaction diffusion equations with nonlocal boundary conditions. J. Math. Anal. Appl. 470, 1-15 (2019)

19. Fang, Z.B., Ma, L.W.: Blow-up analysis for a reaction-diffusion equation with weighted nonlocal inner absorptions under nonlinear boundary flux. Nonlinear Anal., Real World Appl. 32, 338-354 (2016)

20. Liu, Z.Q., Fang, Z.B.: Blow-up phenomena for a nonlocal quasilinear parabolic equations with time-dependent coefficients under nonlinear boundary flux. Discrete Contin. Dyn. Syst., Ser. B 21, 3619-3635 (2016)

21. Enache, C.: Blow-up phenomena for a class of quasilinear parabolic problems under Robin boundary condition. Appl. Math. Lett. 24, 288-292 (2011)

22. Li, Y.F., Liu, Y., Lin, C.H.: Blow-up phenomena for some nonlinear parabolic problems under mixed boundary condition. Nonlinear Anal., Real World Appl. 11, 3815-3823 (2010)

23. Ding, J.T., Shen, X.H.: Blow-up analysis for a class of nonlinear reaction diffusion equations with Robin boundary conditions. Math. Methods Appl. Sci. 41, 1683-1696 (2018)

24. Ding, J.T., Shen, X.H.: Blow-up analysis in quasilinear reaction-diffusion problems with weighted nonlocal source. Comput. Math. Appl. 75(4), 1288-1301 (2018)

25. Sperb, R.P.: Maximum Principles and Their Applications. Academic Press, New York (1981)

\section{Submit your manuscript to a SpringerOpen ${ }^{\circ}$} journal and benefit from:

- Convenient online submission

- Rigorous peer review

- Open access: articles freely available online

- High visibility within the field

- Retaining the copyright to your article

Submit your next manuscript at $\boldsymbol{~ s p r i n g e r o p e n . c o m ~}$ 